\title{
Data Security Using Multi-bit LSB and Modified Vernam Cipher
}

\section{G T Simbolon'1, Opim Salim Sitompul ${ }^{2}$, E B Nababan ${ }^{3}$}

\author{
${ }^{1}$ Graduate School of Computer Science \\ ${ }^{2,3}$ Department of Information Technology, Faculty of Computer Science and Information \\ Technology, Universitas Sumatera Utara, Medan, Indonesia
}

\begin{abstract}
LSB is a steganographic algorithm that is often used to store data in the last bit. Vernam is one of the most popular methods used to encrypt messages easily and quickly. But some possibilities can make LSB and Vernam less safe to use in the long run. Modification of Vernam are made to the bits of each character, the rotation by a certain amount can randomi ze the plaintext content before Vernam encryption is performed. While modifications to the LSB can be performed on some multi-bit models. Bit on LSB can be inserted data as much as 1, 2, 3 or 4-bit information. Based on PSNR and MSE values achieved, 1-bit LSB is superior compare to 2, 3 or 4-bit information.
\end{abstract}

Keyword: Cryptography, LSB, Steganographic, Vernam Encryption

\begin{abstract}
Abstrak. LSB adalah algoritma steganografi yang sering digunakan untuk menyimpan data pada bit terakhir. Vernam adalah salah satu metode yang sangat populer digunakan untuk mengenkripsi pesan dengan mudah dan cepat. Tetapi beberapa kemungkinan dapat menjadikan LSB dan Vernam tidak begitu aman digunakan dalam waktu jangka panjang.. Modifikasi Vernam dilakukan pada susunan bit tiap karakter, rotasi dengan jumlah terentu dapat mengacak isi plaintext sebelum dilakukan enkripsi Vernam. Sementara itu modifikasi pada LSB dapat dilakukan pada beberapa model multi-bit. Bit pada LSB dapat disisip data sebanyak 1, 2, 3 atau 4-bit informasi. Berdasarkan nilai PSNR dan MSE yang didapat, 1-bit LSB lebih baik dibandingkan dengan informasi 2, 3 dan 4 bit.
\end{abstract}

Kata Kunci: Cryptography, LSB, Steganographic, Vernam Encryption

Received date month year. | Revised date month year | Accepted date month year

\section{Introduction}

According to the Cisco Visual Networking Index (VNI), the total IP traffic that occurred in 2016 is expected to reach 91.3 exabytes per month. With the amount of traffic this big, the security of data becomes very important. Especially for sensitive data such as enterprise data and information relating to the security of the State [1]. Digital information will be transmitted over the data network indirectly through a small electrical current used as a link to analog signals [2]. Data is very important information that must be secured properly in order to avoid data theft by certain

\footnotetext{
*Corresponding author at: Department of Information Technology, Faculty of Computer Science and Information Technology, Universitas Sumatera Utara, Medan, Indonesia

E-mail address: ernabrn@usu.ac.id
} 
parties. Steganography and cryptography are two ways that can be used to secure data and have each way. Steganography performs data hiding, while cryptography performs data security by encrypting plaintext. The crime is growing so that both ways often conceded. Various acts of data theft are done to solve the ciphertext that has been well encrypted. This results in steganographic and cryptographic forces to be enhanced in various ways.

The weakness of this LSB technique is the storage of plaintext bits in a row helps intruders to extract bits of confidential information easily. In this we will improve data security by combining steganography and cryptographic techniques. Vernam algorithm encrypts data by performing XOR operations on each plaintext character by rotating bits. Then the ciphertext will be hidden by the LSB steganography technique on 24-bit imagery by comparing several storage bit models. LSB will hide each bit of information into RGB color elements by creating variations of the LSB technique [2] - [5]. Multi-bits can be determined based on the ability of the image to store the ciphertext into the image. We then calculate MSE (Mean Squared Error) and PSNR (Peak Signal to Noise Ratio) which method obtain the least error rate [6], [7] . The method with least MSE value will improve data security.

\section{Methodology}

\subsection{Modification of Vernam Chiper}

In this section we will explain how the Vernam algorithm is modified using bit rotation. During the encryption process the character bit rotation will be left to the left and the key bit rotation to the right. Bit rotation will be done before the XOR operation is performed. When plaintext and the key is given, it will then rotate the position of the 1st to the 8th bit. During the decryption process, the first thing to do is XOR operation between the ciphertext and the key (which has been done right bit rotation), the XOR results are then rotated to the right so that the message returns to its original position (plaintext). Process of modifying Vernam's encryption algorithm is as follow:

Plaintext:

\begin{tabular}{|l|l|l|l|l|}
\hline P0 & P1 & P2 & P3 & P4 \\
\hline
\end{tabular}

Bit Plaintext:

\begin{tabular}{|c|c|c|c|c|c|c|c|c|}
\hline $\mathrm{B} 07$ & B06 & B05 & B04 & $\mathrm{B} 03$ & $\mathrm{~B} 02$ & B01 & $\mathrm{B} 00$ & $\mathrm{PO}$ \\
\hline B17 & B16 & B15 & B14 & B13 & B12 & B11 & B10 & P1 \\
\hline B27 & B26 & B25 & B24 & B23 & B22 & B21 & B20 & P2 \\
\hline B37 & B36 & B35 & B34 & B33 & B32 & B31 & B30 & P3 \\
\hline B47 & B46 & B45 & B44 & B43 & B42 & B41 & B40 & P4 \\
\hline
\end{tabular}


1-bit Rotation to Left:

\begin{tabular}{|c|c|c|c|c|c|c|c|c|}
\hline B06 & B05 & $\mathrm{B} 04$ & $\mathrm{~B} 03$ & $\mathrm{~B} 02$ & B01 & $\mathrm{BOO}$ & $\mathrm{B} 07$ & $\mathrm{PO}$ \\
\hline B16 & B15 & B14 & B13 & B12 & B11 & B10 & B17 & $\mathrm{P} 1$ \\
\hline B26 & B25 & B24 & B23 & B22 & B21 & B20 & B27 & $\mathrm{P} 2$ \\
\hline B36 & B35 & B34 & B33 & B32 & B31 & B30 & B37 & $\mathrm{P3}$ \\
\hline B46 & B45 & B44 & B43 & B42 & B41 & B40 & B47 & P4 \\
\hline
\end{tabular}

Key:

\begin{tabular}{|l|l|l|l|l|}
\hline $\mathrm{K} \theta$ & $\mathrm{K} 1$ & $\mathrm{~K} 2$ & $\mathrm{~K} 3$ & $\mathrm{~K} 4$ \\
\hline
\end{tabular}

Bit Key:

\begin{tabular}{|c|c|c|c|c|c|c|c|}
\hline $\mathrm{K} 07$ & $\mathrm{~K} 06$ & $\mathrm{~K} 05$ & $\mathrm{~K} 04$ & $\mathrm{~K} 03$ & $\mathrm{~K} 02$ & $\mathrm{~K} 01$ & $\mathrm{~K} 00$ \\
\hline $\mathrm{K} 17$ & $\mathrm{~K} 16$ & $\mathrm{~K} 15$ & $\mathrm{~K} 14$ & $\mathrm{~K} 13$ & $\mathrm{~K} 12$ & $\mathrm{~K} 11$ & $\mathrm{~K} 10$ \\
\hline $\mathrm{K} 27$ & $\mathrm{~K} 26$ & $\mathrm{~K} 25$ & $\mathrm{~K} 24$ & $\mathrm{~K} 23$ & $\mathrm{~K} 22$ & $\mathrm{~K} 21$ & $\mathrm{~K} 20$ \\
\hline $\mathrm{K} 37$ & $\mathrm{~K} 36$ & $\mathrm{~K} 35$ & $\mathrm{~K} 34$ & $\mathrm{~K} 33$ & $\mathrm{~K} 32$ & $\mathrm{~K} 31$ & $\mathrm{~K} 30$ \\
\hline $\mathrm{K} 47$ & $\mathrm{~K} 46$ & $\mathrm{~K} 45$ & $\mathrm{~K} 44$ & $\mathrm{~K} 43$ & $\mathrm{~K} 42$ & $\mathrm{~K} 41$ & $\mathrm{~K} 40$ \\
\cline { 4 - 6 } & $\rightarrow$ & $\mathrm{K} 1$ \\
\hline $\mathrm{K} 2$ \\
\hline $\mathrm{K} 3$ \\
$\mathrm{~K} 4$ \\
\hline
\end{tabular}

1-bit rotation to Right:

\begin{tabular}{|c|c|c|c|c|c|c|c|}
\hline $\mathrm{K} 00$ & $\mathrm{~K} 07$ & $\mathrm{~K} 06$ & $\mathrm{~K} 05$ & $\mathrm{~K} 04$ & $\mathrm{~K} 03$ & $\mathrm{~K} 02$ & $\mathrm{~K} 01$ \\
\hline $\mathrm{K} 10$ & $\mathrm{~K} 17$ & $\mathrm{~K} 16$ & $\mathrm{~K} 15$ & $\mathrm{~K} 14$ & $\mathrm{~K} 13$ & $\mathrm{~K} 12$ & $\mathrm{~K} 11$ \\
\hline $\mathrm{K} 20$ & $\mathrm{~K} 27$ & $\mathrm{~K} 26$ & $\mathrm{~K} 25$ & $\mathrm{~K} 24$ & $\mathrm{~K} 23$ & $\mathrm{~K} 22$ & $\mathrm{~K} 21$ \\
\hline $\mathrm{K} 30$ & $\mathrm{~K} 37$ & $\mathrm{~K} 36$ & $\mathrm{~K} 35$ & $\mathrm{~K} 34$ & $\mathrm{~K} 33$ & $\mathrm{~K} 32$ & $\mathrm{~K} 31$ \\
\hline $\mathrm{K} 40$ & $\mathrm{~K} 47$ & $\mathrm{~K} 46$ & $\mathrm{~K} 45$ & $\mathrm{~K} 44$ & $\mathrm{~K} 43$ & $\mathrm{~K} 42$ & $\mathrm{~K} 41$ \\
\hline
\end{tabular}

Ciphertext / XOR Operation:

\begin{tabular}{|l|l|l|l|l|l|l|l|}
\hline $\mathrm{B} 06 \oplus \mathrm{K} 00$ & $\mathrm{~B} 05 \oplus \mathrm{K} 07$ & $\mathrm{~B} 04 \oplus \mathrm{K} 06$ & $\mathrm{~B} 03 \oplus \mathrm{K} 05$ & $\mathrm{~B} 02 \oplus \mathrm{K} 04$ & $\mathrm{~B} 01 \oplus \mathrm{K} 03$ & $\mathrm{~B} 00 \oplus \mathrm{K} 02$ & $\mathrm{~B} 07 \oplus \mathrm{K} 01$ \\
\hline $\mathrm{B} 16 \oplus \mathrm{K} 10$ & $\mathrm{~B} 15 \oplus \mathrm{K} 17$ & $\mathrm{~B} 14 \oplus \mathrm{K} 16$ & $\mathrm{~B} 13 \oplus \mathrm{K} 15$ & $\mathrm{~B} 12 \oplus \mathrm{K} 14$ & $\mathrm{~B} 11 \oplus \mathrm{K} 13$ & $\mathrm{~B} 10 \oplus \mathrm{K} 12$ & $\mathrm{~B} 17 \oplus \mathrm{K} 11$ \\
\hline $\mathrm{B} 26 \oplus \mathrm{K} 20$ & $\mathrm{~B} 25 \oplus \mathrm{K} 27$ & $\mathrm{~B} 24 \oplus \mathrm{K} 26$ & $\mathrm{~B} 23 \oplus \mathrm{K} 25$ & $\mathrm{~B} 22 \oplus \mathrm{K} 24$ & $\mathrm{~B} 22 \oplus \mathrm{K} 23$ & $\mathrm{~B} 20 \oplus \mathrm{K} 22$ & $\mathrm{~B} 27 \oplus \mathrm{K} 21$ \\
\hline $\mathrm{B} 36 \oplus \mathrm{K} 30$ & $\mathrm{~B} 35 \oplus \mathrm{K} 37$ & $\mathrm{~B} 34 \oplus \mathrm{K} 36$ & $\mathrm{~B} 33 \oplus \mathrm{K} 35$ & $\mathrm{~B} 32 \oplus \mathrm{K} 34$ & $\mathrm{~B} 31 \oplus \mathrm{K} 33$ & $\mathrm{~B} 30 \oplus \mathrm{K} 32$ & $\mathrm{~B} 37 \oplus \mathrm{K} 31$ \\
\hline $\mathrm{B} 46 \oplus \mathrm{K} 40$ & $\mathrm{~B} 45 \oplus \mathrm{K} 47$ & $\mathrm{~B} 44 \oplus \mathrm{K} 46$ & $\mathrm{~B} 43 \oplus \mathrm{K} 45$ & $\mathrm{~B} 42 \oplus \mathrm{K} 44$ & $\mathrm{~B} 41 \oplus \mathrm{K} 43$ & $\mathrm{~B} 40 \oplus \mathrm{K} 42$ & $\mathrm{~B} 47 \oplus \mathrm{K} 41$ \\
$\mathrm{C} 1$ \\
$\longrightarrow$
\end{tabular}

Therfore the process of modifying Vernam's decryption algorithm: 
Ciphertext:

\begin{tabular}{|l|l|l|l|l|}
\hline $\mathrm{C} \theta$ & $\mathrm{C} 1$ & $\mathrm{C} 2$ & $\mathrm{C} 3$ & $\mathrm{C} 4$ \\
\hline
\end{tabular}

\subsection{Multi-bit LSB}

Multi-bit LSB is done with several bit insertion models. Following are the steps carried out in the multi-bit LSB process, by inserting messages in specified bits on three elements of color (Red, Green and Blue) in each pixel.

Character storage on 1-bit LSB:

\begin{tabular}{|c|c|c|c|c|c|c|c|c|}
\hline$R$ & 7 & 6 & 5 & 4 & 3 & 2 & 1 & 0 \\
\hline$G$ & 7 & 6 & 5 & 4 & 3 & 2 & 1 & 0 \\
\hline$B$ & 7 & 6 & 5 & 4 & 3 & 2 & 1 & 0 \\
\hline$R$ & 7 & 6 & 5 & 4 & 3 & 2 & 1 & 0 \\
\hline$G$ & 7 & 6 & 5 & 4 & 3 & 2 & 1 & 0 \\
\hline$B$ & 7 & 6 & 5 & 4 & 3 & 2 & 1 & 0 \\
\hline$R$ & 7 & 6 & 5 & 4 & 3 & 2 & 1 & 0 \\
\hline$G$ & 7 & 6 & 5 & 4 & 3 & 2 & 1 & 0 \\
\hline
\end{tabular}

Character storage on 2-bits LSB:

\begin{tabular}{|c|c|c|c|c|c|c|c|c|}
\hline$R$ & 7 & 6 & 5 & 4 & 3 & 2 & 1 & 0 \\
\hline$G$ & 7 & 6 & 5 & 4 & 3 & 2 & 1 & 0 \\
\hline$B$ & 7 & 6 & 5 & 4 & 3 & 2 & 1 & 0 \\
\hline$R$ & 7 & 6 & 5 & 4 & 3 & 2 & 1 & 0 \\
\hline
\end{tabular}

Character storage on 3-bits LSB:

\begin{tabular}{|c|c|c|c|c|c|c|c|c|}
\hline $\mathrm{R}$ & 7 & 6 & 5 & 4 & 3 & 2 & 1 & 0 \\
\hline $\mathrm{G}$ & 7 & 6 & 5 & 4 & 3 & 2 & 1 & 0 \\
\hline $\mathrm{B}$ & 7 & 6 & 5 & 4 & 3 & 2 & 1 & 0 \\
\hline
\end{tabular}

Character storage on 4-bits LSB:

\begin{tabular}{|c|c|c|c|c|c|c|c|c|}
\hline$R$ & 7 & 6 & 5 & 4 & 3 & 2 & 1 & 0 \\
\hline$G$ & 7 & 6 & 5 & 4 & 4 & 2 & 1 & 0 \\
\hline
\end{tabular}

The four patterns above save as much as 1 character ( 8 bits). The more bits used, the less number of pixels used as information storage. 1-bit storage uses less than 3 pixels, on 2-bit storage using less than 2 pixels, 3-bit storage uses exactly 1 pixel and 4-bit storage also uses 1 pixel. In empty 
color elements can be stored for the next character or can be skipped and restarted on the next color pixel element, which is Red.

\subsection{Calculation of MSE and PSNR}

The image used is 24-bit color image. The calculation of MSE and PSNR aims to determine how much the image changes after message insertion. There is 1-bit storage up to 4-bit LSB to be performed, each stego-image will be calculated MSE and PSNR values to determine which image is better or how many better bits to store information or messages. The following is the formula used to calculate MSE and PSNR.

$M S E=\sum_{i=1}^{M} \sum_{j=1}^{N}(f(i, j)-g(i, j))^{2} / M * N$

Information:

$$
\begin{aligned}
& \text { MSE } \quad \text { The MSE value of image } \\
& \mathrm{M} \quad \text { = Image of length } \\
& \mathrm{N} \quad \text { = Image of width } \\
& \mathrm{f}(\mathrm{i}, \mathrm{j}) \quad=\text { The pixel coordinate value of the image before the message is inserted } \\
& \mathrm{g}(\mathrm{i}, \mathrm{j}) \quad=\text { The pixel coordinate value of the image after the message is inserted }
\end{aligned}
$$

Example:

Table 3.1 RGB Pixel

$$
\begin{aligned}
& \begin{array}{|l|l|l|}
\hline \multicolumn{3}{|c|}{\text { Red }} \\
\hline \text { R1 } & \cdots & \mathrm{Rn} \\
\hline
\end{array} \\
& \begin{array}{|l|l|l|}
\hline \multicolumn{3}{|c|}{\text { Green }} \\
\hline \text { G1 } & \cdots & \text { Gn } \\
\hline
\end{array} \\
& \begin{array}{|l|l|l|}
\hline \multicolumn{3}{|c|}{\text { Blue }} \\
\hline \text { B1 } & \cdots & \text { Bn } \\
\hline
\end{array} \\
& \mathrm{MSE}=\frac{1}{M * N} \\
& \left(\frac{\left(R_{1}^{\prime}-R_{1}\right)^{2}+\cdots+\left(R_{n}^{\prime}-R_{n}\right)^{2}+\left(G_{1}^{\prime}-G_{1}\right)^{2}+\cdots+\left(G_{n}^{\prime}-G_{n}\right)^{2}+\left(B_{1}^{\prime}-B_{1}\right)^{2}+\cdots+\left(B_{n}^{\prime}-B_{n}\right)^{2}}{3}\right) \\
& P S N R=10 \log _{10}\left(\frac{C_{\max }^{2}}{M S E}\right)
\end{aligned}
$$

Information:

PSNR = The PSNR value of image

$C^{2}{ }_{\max }=$ The highest pixels of the RGB image 


\section{Result and Discussion}

This section will test the success of combining steganography and cryptography with modifications made. This test with 1-bit rotation and determines the MSE and PNSR values of each stego-image. Examples of images used are $4 \times 4$ pixels. This image can accommodate as many as 48-bits. If converted to characters, it can accommodate as many as 6 characters for the use of 1 bit. The following tests are carried out on this method.

Plaintext : Secret

\begin{tabular}{|l|l|l|l|l|l|}
\hline 83 & 101 & 99 & 114 & 101 & 116 \\
\hline 01010011 & 01100101 & 01100011 & 01110010 & 01100101 & 01110100 \\
\hline
\end{tabular}

Rotation : 1-bit to the left

\begin{tabular}{|l|l|l|l|l|l|}
\hline 10100110 & 11001010 & 11000110 & 11100100 & 11001010 & 11101000 \\
\hline 166 & 202 & 198 & 228 & 202 & 232 \\
\hline
\end{tabular}

Key : World

\begin{tabular}{|l|l|l|l|l|}
\hline 87 & 111 & 114 & 108 & 100 \\
\hline 01010111 & 01101111 & 01110010 & 01101100 & 01100100 \\
\hline
\end{tabular}

Rotation : 1-bit to the right

\begin{tabular}{|l|l|l|l|l|}
\hline 10101011 & 10110111 & 00111001 & 00110110 & 00110010 \\
\hline 171 & 183 & 57 & 54 & 50 \\
\hline
\end{tabular}

Ciphertext : -$\} \ddot{\mathrm{y}} \hat{\mathrm{E}}^{\circ} \mathrm{C}$

\begin{tabular}{|l|l|l|l|l|l|}
\hline 13 & 125 & 255 & 210 & 248 & 67 \\
\hline
\end{tabular}

The results of the ciphertext will be stored in an image with several bit storage models, such as the following illustration. In this test, an image piece of $4 \times 4$ pixels will be taken as a cover image. The capacity that can be accommodated by this image is $4 \times 4 \times 3$ bits $=48$ bits $(6$ characters) on the use of 1 bit.

RGB bit for the $4 \times 4$ pixel cover image:

\begin{tabular}{|c|c|c|c|}
\hline \multicolumn{4}{|c|}{ Red } \\
\hline 65 & 58 & 187 & 190 \\
\hline 222 & 171 & 203 & 63 \\
\hline
\end{tabular}

\begin{tabular}{|c|c|c|c|}
\hline \multicolumn{4}{|c|}{ Green } \\
\hline 87 & 176 & 149 & 241 \\
\hline 223 & 74 & 221 & 68 \\
\hline
\end{tabular}

\begin{tabular}{|l|l|l|l|}
\hline \multicolumn{4}{|c|}{ Blue } \\
\hline 116 & 249 & 167 & 229 \\
\hline 150 & 134 & 191 & 182 \\
\hline
\end{tabular}




\begin{tabular}{|l|l|l|l|}
246 & 101 & 32 & 89 \\
\hline 192 & 151 & 36 & 11 \\
\hline
\end{tabular}

\begin{tabular}{|l|l|l|l|}
205 & 204 & 143 & 53 \\
\hline 125 & 130 & 167 & 60 \\
\hline
\end{tabular}

\begin{tabular}{|l|l|l|l|}
128 & 84 & 243 & 163 \\
\hline 226 & 101 & 153 & 23 \\
\hline
\end{tabular}

The ciphertext bit that will be inserted: $\quad-\} \ddot{\mathrm{y}} \hat{\mathrm{E}}^{\circ} \mathrm{C}$

\begin{tabular}{|l|l|l|l|l|l|}
\hline 00001101 & 01111101 & 11111111 & 11010010 & 11111000 & 01000011 \\
\hline
\end{tabular}

Stego-image results on 1-bit LSB:

\begin{tabular}{|c|c|c|c|}
\hline \multicolumn{5}{|c|}{ Red } \\
\hline$\underline{\mathbf{6 4}}$ & 58 & $\underline{\mathbf{1 8 6}}$ & $\underline{\mathbf{1 9 1}}$ \\
\hline$\underline{\mathbf{2 2 3}}$ & 171 & 203 & 63 \\
\hline$\underline{\mathbf{2 4 7}}$ & 101 & $\underline{\mathbf{3 3}}$ & 89 \\
\hline$\underline{\mathbf{1 9 3}}$ & $\underline{\mathbf{1 5 0}}$ & 36 & $\underline{\mathbf{1 0}}$ \\
\hline
\end{tabular}

\begin{tabular}{|c|c|c|c|}
\hline \multicolumn{5}{|c|}{ Green } \\
\hline$\underline{\mathbf{8 6}}$ & $\underline{\mathbf{1 7 7}}$ & 149 & 241 \\
\hline 223 & $\underline{\mathbf{7 5}}$ & 221 & $\underline{\mathbf{6 9}}$ \\
\hline 205 & 204 & $\underline{\mathbf{1 4 2}}$ & 53 \\
\hline$\underline{\mathbf{1 2 4}}$ & 130 & $\underline{\mathbf{1 6 6}}$ & $\underline{\mathbf{6 1}}$ \\
\hline
\end{tabular}

\begin{tabular}{|c|c|c|c|}
\hline \multicolumn{4}{|c|}{ Blue } \\
\hline 116 & 249 & $\underline{\mathbf{1 6 6}}$ & 229 \\
\hline 150 & $\underline{\mathbf{1 3 5}}$ & 191 & $\underline{\mathbf{1 8 3}}$ \\
\hline 128 & 84 & 243 & 163 \\
\hline 226 & 101 & $\underline{\mathbf{1 5 2}}$ & 23 \\
\hline
\end{tabular}

$$
\begin{aligned}
\operatorname{MSE} & =\frac{1}{16}(7) \\
& =0,4375 \\
\text { PSNR } & =10 * \log 10\left(\frac{249^{2}}{0,4375}\right) \\
& =51,5142
\end{aligned}
$$

Stego-image results on 2-bit LSB:

\begin{tabular}{|c|c|c|c|}
\hline \multicolumn{5}{|c|}{ Red } \\
\hline$\underline{\mathbf{6 4}}$ & $\underline{\mathbf{5 7}}$ & 187 & $\underline{\mathbf{1 9 1}}$ \\
\hline$\underline{\mathbf{2 2 3}}$ & $\underline{\mathbf{1 7 0}}$ & $\underline{\mathbf{2 0 2}}$ & $\underline{\mathbf{6 0}}$ \\
\hline 246 & 101 & 32 & 89 \\
\hline 192 & 151 & 36 & 11 \\
\hline
\end{tabular}

\begin{tabular}{|c|c|c|c|}
\hline \multicolumn{5}{|c|}{ Green } \\
\hline$\underline{\mathbf{8 4}}$ & $\underline{\mathbf{1 7 7}}$ & 149 & $\underline{\mathbf{2 4 3}}$ \\
\hline$\underline{\mathbf{2 2 1}}$ & $\underline{\mathbf{7 5}}$ & $\underline{\mathbf{2 2 0}}$ & 68 \\
\hline 205 & 204 & 143 & 53 \\
\hline 125 & 130 & 167 & 60 \\
\hline
\end{tabular}

\begin{tabular}{|c|c|c|c|}
\hline \multicolumn{5}{|c|}{ Blue } \\
\hline$\underline{\mathbf{1 1 9}}$ & $\underline{\mathbf{2 5 1}}$ & 167 & $\underline{\mathbf{2 3 1}}$ \\
\hline$\underline{\mathbf{1 4 8}}$ & $\underline{\mathbf{1 3 5}}$ & $\underline{\mathbf{1 8 9}}$ & $\underline{\mathbf{1 8 3}}$ \\
\hline 128 & 84 & 243 & 163 \\
\hline 226 & 101 & 153 & 23 \\
\hline
\end{tabular}

$$
\begin{aligned}
\operatorname{MSE} & =\frac{1}{16}(10,67) \\
& =0,6667 \\
\text { PSNR } & =10 * \log 10\left(\frac{249^{2}}{0,6667}\right) \\
& =49,6846
\end{aligned}
$$


Stego-image results on 3-bit LSB:

\begin{tabular}{|c|c|c|c|}
\hline \multicolumn{5}{|c|}{ Red } \\
\hline$\underline{\mathbf{6 4}}$ & $\underline{\mathbf{6 3}}$ & $\underline{\mathbf{1 9 1}}$ & $\underline{\mathbf{1 8 8}}$ \\
\hline$\underline{\mathbf{2 2 0}}$ & 171 & 203 & 63 \\
\hline 246 & 101 & 32 & 89 \\
\hline 192 & 151 & 36 & 11 \\
\hline
\end{tabular}

\begin{tabular}{|c|c|c|c|}
\hline \multicolumn{5}{|c|}{ Green } \\
\hline$\underline{\mathbf{8 3}}$ & $\underline{\mathbf{1 8 2}}$ & $\underline{\mathbf{1 5 1}}$ & $\underline{\mathbf{2 4 6}}$ \\
\hline$\underline{\mathbf{2 1 8}}$ & 74 & 221 & 68 \\
\hline 205 & 204 & 143 & 53 \\
\hline 125 & 130 & 167 & 60 \\
\hline
\end{tabular}

\begin{tabular}{|c|c|c|c|}
\hline \multicolumn{5}{|c|}{ Blue } \\
\hline$\underline{\mathbf{1 1 3}}$ & $\underline{\mathbf{2 5 5}}$ & $\underline{\mathbf{1 6 6}}$ & $\underline{\mathbf{2 3 1}}$ \\
\hline$\underline{\mathbf{1 4 4}}$ & 134 & 191 & 182 \\
\hline 128 & 84 & 243 & 163 \\
\hline 226 & 101 & 153 & 23 \\
\hline
\end{tabular}

$$
\begin{aligned}
\operatorname{MSE} & =\frac{1}{16}(18) \\
& =1,125 \\
\text { PSNR } & =10 * \log 10\left(\frac{249^{2}}{1,125}\right) \\
& =47,4142
\end{aligned}
$$

Stego-image results on 4-bit LSB:

\begin{tabular}{|c|c|c|c|}
\hline \multicolumn{5}{|c|}{ Red } \\
\hline$\underline{\mathbf{6 4}}$ & $\underline{\mathbf{6 1}}$ & $\underline{\mathbf{1 8 9}}$ & $\underline{\mathbf{1 8 4}}$ \\
\hline 222 & 171 & 203 & 63 \\
\hline 246 & 101 & 32 & 89 \\
\hline 192 & 151 & 36 & 11 \\
\hline
\end{tabular}

\begin{tabular}{|c|c|c|c|}
\hline \multicolumn{5}{|c|}{ Green } \\
\hline$\underline{\mathbf{9 3}}$ & $\underline{\mathbf{1 9 1}}$ & $\underline{\mathbf{1 4 6}}$ & $\underline{\mathbf{2 4 4}}$ \\
\hline 223 & 74 & 221 & 68 \\
\hline 205 & 204 & 143 & 53 \\
\hline 125 & 130 & 167 & 60 \\
\hline
\end{tabular}

\begin{tabular}{|c|c|c|c|}
\hline \multicolumn{5}{|c|}{ Blue } \\
\hline$\underline{\mathbf{1 1 9}}$ & $\underline{\mathbf{2 5 5}}$ & $\underline{\mathbf{1 7 5}}$ & $\underline{\mathbf{2 2 7}}$ \\
\hline 150 & 134 & 191 & 182 \\
\hline 128 & 84 & 243 & 163 \\
\hline 226 & 101 & 153 & 23 \\
\hline
\end{tabular}

$$
\begin{aligned}
\operatorname{MSE} & =\frac{1}{16}(19,333) \\
& =1,2083 \\
\text { PSNR } & =10 * \log 10\left(\frac{249^{2}}{1,2083}\right) \\
& =47,1022
\end{aligned}
$$

Information:

Bold and underlined pixel bits are pixel bits that have been inserted into the message. In 1-bit LSB storage more image pixels are used because each RGB color element can only store 1-bit. For storing 4-bit LSB fewer pixels are used because each RGB color element can store 4-bit ciphertext. 


\section{Conclusion}

The level of data security is increased by combining steganographic techniques with cryptographic algorithms. Image resolution for the cover image and character length of the message, greatly affect the value of the MSE and PSNR parameters. Seeing the results of the calculation of MSE and PSNR, the use of 1-bit LSB is much better than 2, 3 or 4-bit. This happens because the MSE value in 1-bit is much smaller and also the PSNR value is above $40 \mathrm{db}$. The use of 2-bit can still be considered to obtain good results because the value of MSE is still small and the PSNR still remains above the stipulated value. The implementation of the multi-bit LSB method in steganography activities provides advantages where each pixel can hold more message bits than the usual LSB method.

\section{REFERENCES}

[1] Cisco Visual Networking Index. 2017. Forecast and Methodology. cisco.com, http://www.cisco.com/c/en/us/solutions/collateral/ service-provider/ip-ngn-ip-nextgeneration-network/white_paper_c11-481360.html. [Accessed: 30-Mar-2018]

[2] B. Datta, P. K. Pal, and S. K. Bandyopadhyay, "Multi-bit Data Hiding in Randomly Chosen LSB Layers of an Audio," in 2016 International Conference on Information Technology (ICIT), 2016, pp. 283-287.

[3] M. Kaur and M. Juneja, "A new LSB embedding for 24-bit pixel using multi-layered bitwise XOR," in 2016 International Conference on Inventive Computation Technologies (ICICT), 2016, pp. 1-5.

[4] R.-J. Chen, Y.-C. Chen, Jui-Linlai, and S.-J. Horng, "Data Hiding Using Flexible Multibit MER," in 2013 International Symposium on Biometrics and Security Technologies, 2013, pp. 24-31.

[5] S. Goel, S. Gupta, and N. Kaushik, "Image Steganography - Least Significant Bit with Multiple Progressions," 2015, pp. 105-112.

[6] "The Vernam Cipher," Crypto Museum. [Online]. Available: http://www.cryptomuseum.com/crypto/vernam.htm.

[7] C. . B. S., P. K., and R. D. K., "Least Significant Bit Algorithm for Image Steganography," Int. J. Adv. Comput. Technol., vol. 3, no. 4, pp. 34-38, 2014. 